\title{
Classifying the Phases of Gauge Theories by Spectral Density of Probing Chiral Quarks
}

\author{
Andrei Alexandru \\ The George Washington University, Washington DC, USA \\ E-mail: aalexan@gwu . edu

\section{Ivan Horváth*} \\ University of Kentucky, Lexington, KY, USA \\ E-mail: horvath@pa.uky.edu
}

\begin{abstract}
We describe our recent proposal that distinct phases of gauge theories with fundamental quarks translate into specific types of low-energy behavior in Dirac spectral density. The resulting scenario is built around new evidence substantiating the existence of a phase characterized by bimodal (anomalous) density, and corresponding to deconfined dynamics with broken valence chiral symmetry. We argue that such anomalous phase occurs quite generically in these theories, including in "real world" QCD above the crossover temperature, and in zero-temperature systems with many light flavors.
\end{abstract}

The 33rd International Symposium on Lattice Field Theory

14 -18 July 2015

Kobe International Conference Center, Kobe, Japan

\footnotetext{
* Speaker.
} 

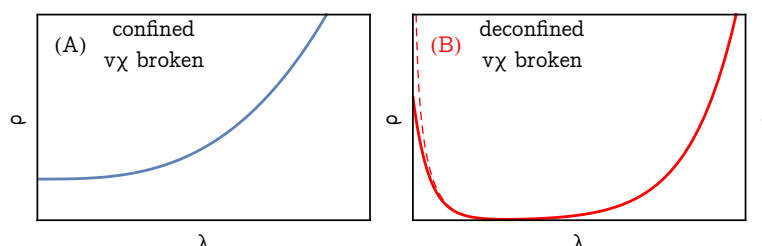
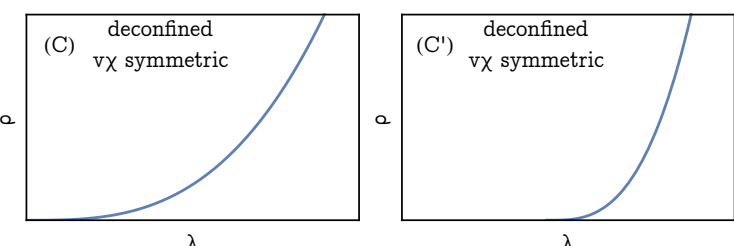

Figure 1: Dirac spectral density and confinement/vSChSB structure of $\mathcal{T}$.

1. Introduction. The phase structure of gauge theories is actively pursued today, mainly due to the heavy ion physics programs at RHIC and LHC, and as a part of BSM searches related to Higgs discovery. Given its relation to strong interactions, $\mathrm{SU}(3)$ is particularly interesting in this regard.

Our take on the phases is motivated, in part, by its potential role in deciphering the vacuum (and thermal state) structure of strong interactions. Given that, we work with more generic notion of a phase than what is typical e.g. in states of matter considerations. Indeed, here it is simply a region of parameter space with definite dynamical property. The phase structure, then, is the induced partition of this space via a chosen collection of such properties. Notice that, if dynamical features in question are well-defined, phase boundaries arise automatically. Transitions mark the corresponding changes of dynamics but are not necessarily associated with singularities.

In this talk, the theory space $\mathcal{T}$ is spanned by $\mathrm{SU}(3)$ gauge theories with fundamental quarks: included are cases with arbitrarily many flavors of any individual masses, and the system can be thermal. We pursue an elementary goal within the above scheme: to design simple dynamical properties so that the resulting phase structure relates to traditional approach of characterizing vacua in terms of confinement and spontaneous chiral symmetry breaking (SChSB) [1].

Our rationale for approaching this is as follows. Consider the gauge vacua/thermal states of all theories contained in $\mathcal{T}$, and let external quarks propagate on the associated backgrounds. If qualitatively different dynamical responses are obtained for two different theories, then these theories entail qualitatively different vacua or thermal states.

As a general strategy, this is hardly new. Indeed, the classic approach to confinement in pure glue case involves infinitely heavy external quark-antiquark pair, and the dependence of their energy on separation. This leads to study of extended gauge observables, Wilson loops, and to a welldefined criterion for confinement in this special case.

However, as is well known, this criterion fails in theories with dynamical quarks. Nevertheless, we intend to keep the idea of probing gauge vacua by external quarks even in general case. The only way to proceed then is to let probing quarks have finite mass and propagate spatially. In fact, we go to the very opposite mass corner, and propose that it is fruitful to probe vacua with quarks that are very light: always much lighter than any physical dynamical quarks native to the theory. ${ }^{1}$

What will reflect the qualitative change in dynamics of these light external quarks? We suggest that the simplest yet powerful indicator of both confinement and SChSB is the infrared end of Dirac spectral density [1]. This crucial element of the proposal needs to be elaborated upon first.

2. Dirac Spectral Density and the Claim. One can easily see why Dirac spectral density is a reasonable guess if one tries to characterize dynamical response of external quarks (field $\eta$ ). After all, Dirac operator defines their dynamics via interaction $\bar{\eta}\left(D+m_{v}\right) \eta$, where $m_{v}$ is the mass of a

\footnotetext{
${ }^{1}$ External quarks will be frequently referred to as "valence quarks", as is common in lattice community.
} 
probing quark as opposed to dynamical quark masses $m_{f}$. Moreover, the Dirac matrix naturally involves scale dependence via its spectrum $D \psi_{\lambda}=i \lambda \psi_{\lambda}$. Thus, to distinguish infrared from ultraviolet, we reduce $D$ to the simplest gauge invariant object retaining this scale dependence. The result is just the Dirac spectral density $\rho(\lambda)$, namely

$$
\rho\left(\lambda, m_{f}, V\right) \equiv \frac{\partial}{\partial \lambda} \sigma\left(\lambda, m_{f}, V\right) \quad \sigma\left(\lambda, m_{f}, V\right) \equiv \frac{1}{V}\left\langle\sum_{0 \leq \lambda_{k}<\lambda} 1\right\rangle_{m_{f}, V}
$$

Note that, in this context, spectral density is treated as a gauge vacuum object assigned to each theory in $\mathcal{T}$. The dynamics of very light external quarks is dominated by infrared part of the Dirac spectrum, which will provide us with needed dynamical signatures.

Another hint in favor of Dirac spectral density is that mode condensation $(\rho(\lambda \rightarrow 0)>0)$ indicates valence chiral symmetry breaking (vSChSB, $\bar{\eta} \eta \neq 0$ ). Strictly infrared limit of $\rho(\lambda)$ is thus already known to contain part of relevant information: gauge vacuum able to support valence condensate is qualitatively different from one that does not.

Given these considerations, our proposal may be represented by Fig.1, indicating that $\mathcal{T}$ is spanned by three behaviors of spectral density [1]. The difference between the two monotonic types, (A) and (C, $\left.\mathrm{C}^{\prime}\right)$, is the zero intercept of the latter. The non-standard non-monotonic case (B) is referred to as anomalous, its relevant feature being the bimodal distribution of modes. Existence of such infrared-ulraviolet separation in response of light probing quarks to the vacuum of a given theory characterizes this vacuum as being deconfined and (valence) chirally broken. Note that there are two types of mode condensation, namely (A) and (B), and thus two types of vSChSB: standard and anomalous. However, the proposal entails only one type of spectral density reflecting confinement: type (A). This classification implies that phase that is simultaneously confined and chirally symmetric doesn't appear.

Finally, important aspect of this proposal is that the anomalous phase (B) is not a rarity in $\mathcal{T}$. Rather, we conclude that it occurs generically along paths connecting standard phases (A) and (C). This dramatically changes the conventional view of phases in theories with fundamental quarks [1].
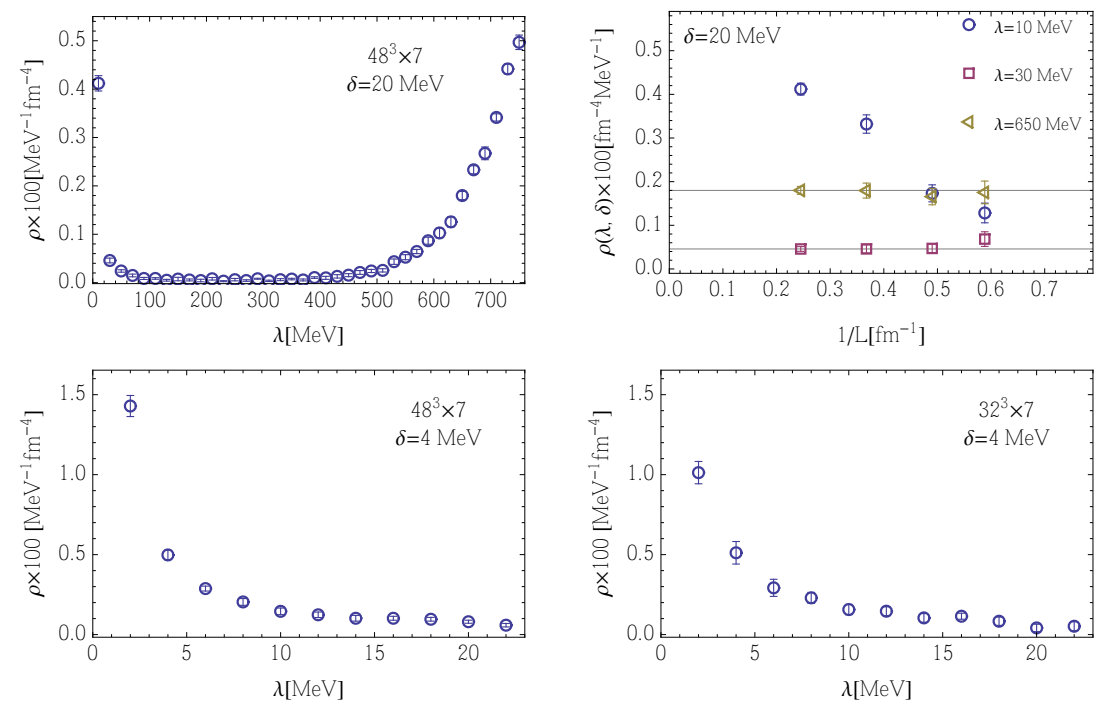

Figure 2: Stability of anomalous phase in thermal $\mathrm{N}_{f}=0$ theory under infrared cutoff. 
3. Existence of the Anomalous Phase and Confinement. The support of the broad picture described above begins by showing its validity at least in some part of $\mathcal{T}$. This can be done in thermal $\mathrm{N}_{f}=0$ theory where both $\mathrm{vSChSB}$ and confinement are well-defined. The situation is particularly clean when overlap fermions define the dynamics of external quarks, which is what we assume from now on. Spectral density of type (B) was first observed in this context some time ago [2], but it was treated as an artifact, its reality not beeing pursued. We thus need to show that the phase is stable under both the infrared (volume) and the ultraviolet (lattice spacing) cutoffs [1].

To do this, Wilson's lattice theory at $\mathrm{T} / \mathrm{T}_{c}=1.12$, set via $r_{0} T_{c}$ [3], was used. We reported bimodality of spectral density at this temperature previously $[4,5]$. The "real Polyakov line" vacuum was always selected in spectral calculations with overlap operator, whose details are described in Ref. [1]. To check infrared stability, we simulated $N^{3} \times 7$ systems at $N=20,24,32,48$ and gauge coupling corresponding to $a=0.085 \mathrm{fm}$ to set the desired temperature. Bimodal spectral density, exemplified by top-left plot of Fig. 2, was obtained at all volumes. Note that $\delta$ is the spectral coarse-graining parameter (bin width).

The density shows very good volume scaling, as seen in the top-right plot. The most infrared bin follows a growing trend though, ensuring that the anomalous shape (B) results in the infinitevolume limit. The bottom-left plot in Fig. 2 shows the closeup on this first bin $(\delta=4 \mathrm{MeV})$, revealing a rapidly decreasing function whose shape again doesn't change with infrared cutoff (bottom-right). Indeed, increasing the volume only makes the bimodal feature more robust. Our conclusion is that bimodal $\rho(\lambda)$ of type (B) is the property of the infinite-volume limit.

Regarding the continuum limit, we fixed the physical volume to that of $N=24$ theory, and increased ultraviolet cutoff while keeping the temperature fixed. This produced lattices $N \times N_{t}=$ $24 \times 7,28 \times 8,34 \times 10,42 \times 12$ with $a=0.0850,0.0744,0.0595,0.0496 \mathrm{fm}$ respectively. Spectral densities were found to be bimodal at all cutoffs. The global view and the closeup at finest lattice spacing are shown in Fig. 3 (top). Varying ultraviolet cutoff should not significantly affect deeply infrared scales unless there is a transition separating lattice and continuum-like behaviors. This is
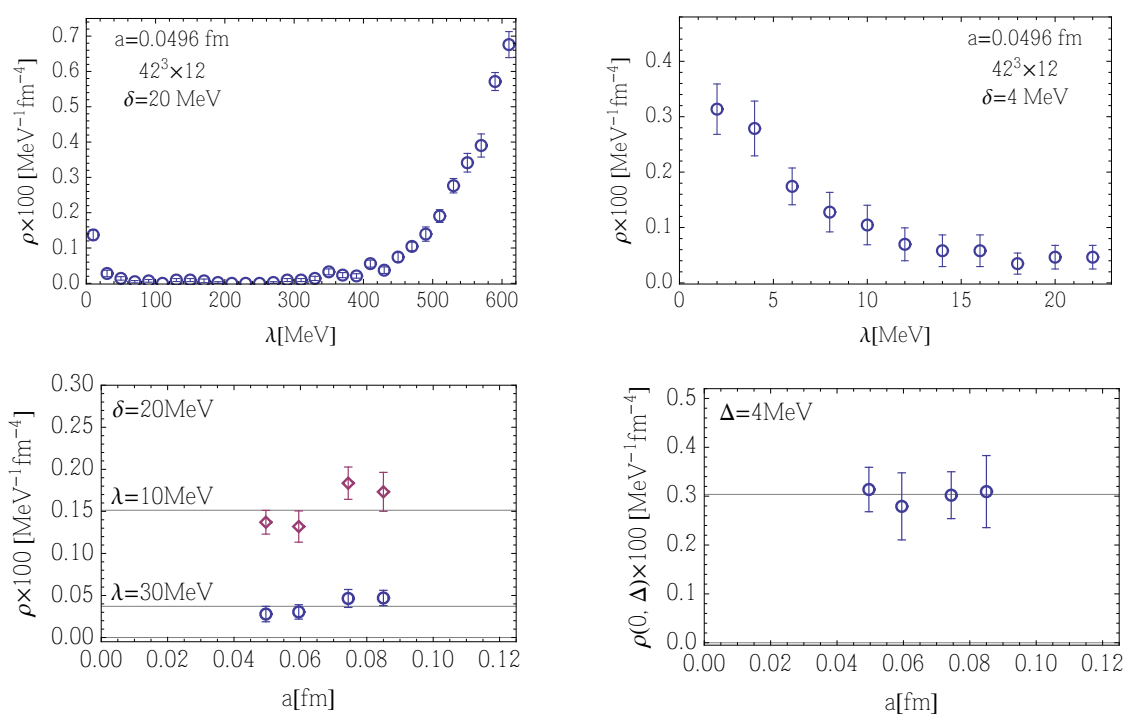

Figure 3: Stability of anomalous phase in thermal $\mathrm{N}_{f}=0$ theory under ultraviolet cutoff. 

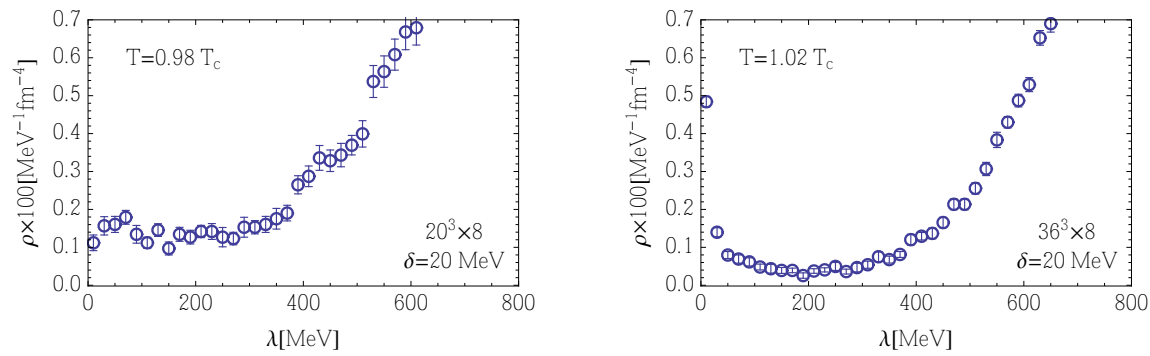

Figure 4: Deconfinement and transition to anomalous phase in $\mathrm{N}_{f}=0$ theory.

not observed as evidenced by lower-left plot showing the scaling of first two bins at $\delta=20 \mathrm{MeV}$ : rather, the results indicate that the bimodal shape will persist into the continuum limit. The estimates of mode condensate from very near-zeromodes i.e. $\rho(0, \Delta) \equiv \sigma(0, \Delta) / \Delta$ with $\Delta=4 \mathrm{MeV}$, are stable as well (lower right), confirming the absence of any qualitative change. These results substantiate the conclusion that continuum $\mathrm{N}_{f}=0$ theory at $T / T_{c}=1.12$ is in the anomalous phase.

Finally, it is necessary to demonstrate the connection of anomalous phase to deconfinement, in this theory i.e. that the transition from (A) to (B) occurs at deconfinement temperature $T_{c}$. To do that, we simulated the system just below and above $T_{c}\left(T=0.98 T_{c}\right.$ and $\left.T=1.02 T_{c}\right)$. The result is shown in Fig. 4, indicating that the onsets of deconfinement and anomalous phase indeed coincide.

4. "Real World" QCD at Finite Temperature. From the physics standpoint it is clearly important to establish whether nature's quarks and gluons can be in the anomalous phase. The vacuum of $\mathrm{N}_{f}=2+1$ QCD at physical quark masses is a very precise representation of the true strong vacuum. The crossover nature of thermal transition in strong interactions was in fact worked out within this framework [6]. To see whether anomalous phase is encountered in the crossover region, we calculated overlap Dirac spectra in the same setting.

Computations were performed on $32^{3} \times 8$ lattices of stout-improved staggered fermions and Symanzik-improved glue (see Ref. [7] for details). Transition temperature is not a unique concept at crossover, and depends on the defining observable. In this case, the temperature from $\bar{\psi} \psi$ was reported to be $T_{c} \approx 155 \mathrm{MeV}$, and from Polyakov line $T_{c} \approx 170 \mathrm{MeV}$. We thus chose ensembles at temperatures $T=150,175$ and $200 \mathrm{MeV}$, covering the crossover region and beyond.

Computed spectral densities are shown in Fig. 5. One can see that just below the lower edge of the crossover (left), $\rho(\lambda)$ exhibits the expected (A) behavior, while at the upper edge (middle),
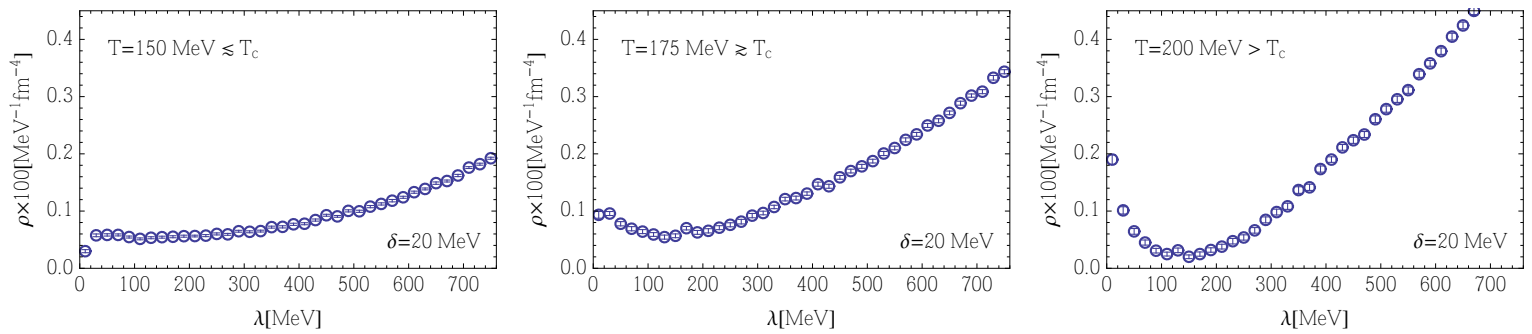

Figure 5: Anomalous phase across the thermal crossover in $\mathrm{N}_{f}=2+1 \mathrm{QCD}$ at physical point. 

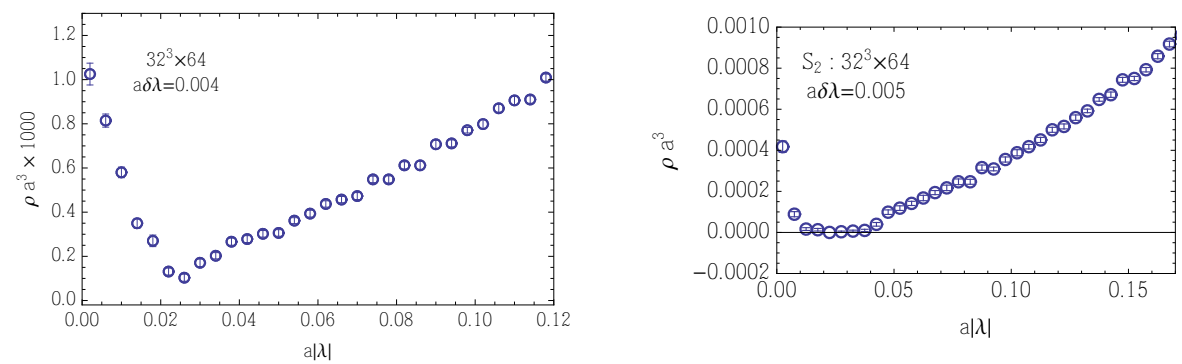

Figure 6: Anomalous spectral densities in $\mathrm{N}_{f}=8$ (left) and $\mathrm{N}_{f}=12$ (right) [5] theories.

the anomalous phase is present. ${ }^{2}$ Heating the system well past the crossover region $(T=200 \mathrm{MeV})$, the bimodal nature of the spectrum completely takes over (right). Since the ensembles used were extensively checked for lattice artifacts, the above evidence leads us to conclude that the anomalous phase $T_{c}<T<T_{c h}$, is a feature of strong interactions, and the proposed general scheme is obeyed. Here $T_{c}$ is defined by the onset of anomalous phase, and $T_{c h}$ marks the valence chiral restoration temperature.

5. Anomalous Phase via Light Quarks. Somewhat surprisingly, it was recently found that the effects of light dynamical quarks alone, without thermal bath, generate anomalous phases at sufficiently large number of flavors [5,9]. Specifically, in $\mathrm{N}_{f}=12$ theory with staggered fermions, it was observed that there is a mass $m_{c}$ below which the anomalous phase appears. The bimodality of Dirac spectrum either extends to arbitrary small masses, or there is a non-zero $m_{c h}<m_{c}$ below which valence chiral symmetry gets restored. In this way, mass effects are analogous to those of temperature.

It is interesting to determine the minimal number of flavors realizing the above scenario. To get the first insight, we made a preliminarily inquiry into the $\mathrm{N}_{f}=8$ theory. As in the $\mathrm{N}_{f}=12$ case, we deal with staggered nHYP dynamical fermions and the fundamental-adjoint combination in gauge action to avoid known lattice artifacts $\left(\beta_{F}=4.8, \beta_{A} / \beta_{F}=-0.25\right)$. The simulation was carried out at zero mass, with finite volume providing for a small effective gap in the staggered spectrum. The result is shown in Fig. 6 (left). Clearly, strong bimodality is observed in this case as well. The anomalous spectrum for $\mathrm{N}_{f}=12\left(\mathrm{am}=0.0025, \beta_{F}=2.4\right)$ is also shown [5], although a well-defined comparison (equivalent volumes, couplings and effective masses) is obviously difficult to arrange.

6. The Generalization. We showed that both light-quark effects (low masses, large $\mathrm{N}_{f}$ ) and thermal effects (large $T$ ) lead to type (B) anomalous phases. However, these are the only available tuning knobs to change the nature of vacuum/thermal state in $\mathcal{T}$. We thus propose that anomalous phases occur generically on paths connecting chirally broken and chirally symmetric vacua. Moreover, the presented evidence for coincidence of anomalous and deconfined/chirally broken phases in relevant parts of $\mathcal{T}$ leads us to extend the correspondence as described in Sec. 2.

The proposed scenario brings in a significant change into the standard picture of $\mathcal{T}$ in terms of chiral symmetry breaking and confinement. Indeed, the contrast can be represented by Fig. 7 . In the new scenario (left) the anomalous phase is wedged between the standard confined/broken and deconfined/symmetric phases (A) and (C). With other parameters fixed, the anomalous regimes

\footnotetext{
${ }^{2}$ Thermal anomalous phase in similar setting was also seen in Ref. [8].
} 

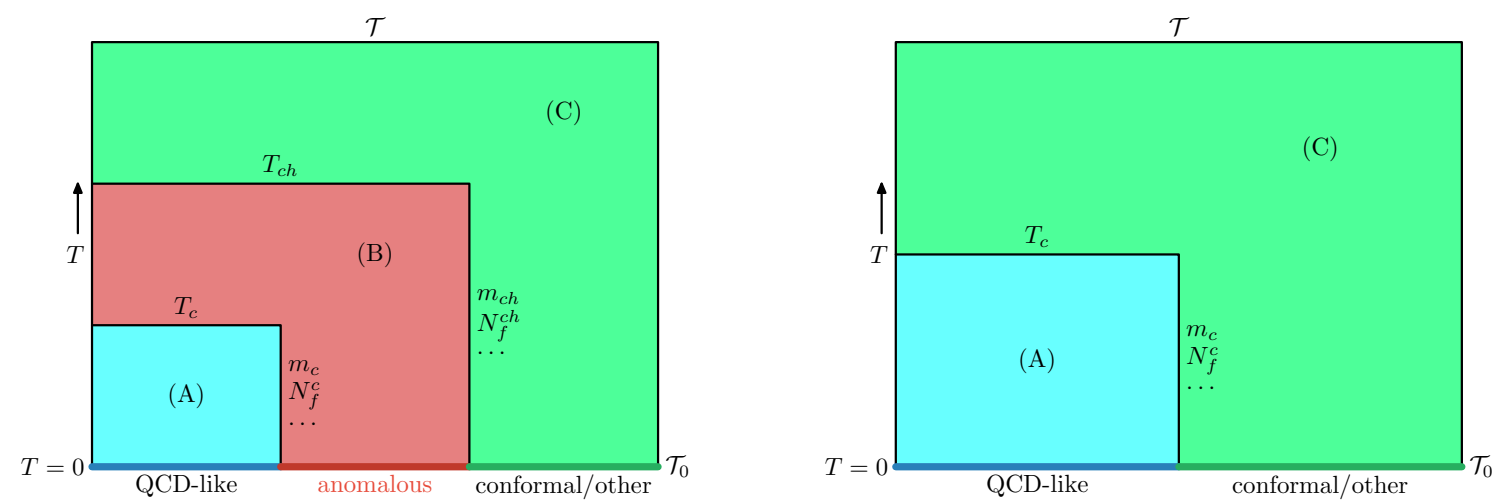

Figure 7: The proposed structure of set $\mathcal{T}$ (left) compared to the conventional one (right).

$T_{c}<T<T_{c h}$ and $m_{c h}<m<m_{c}$ are particular cases of this generic behavior.

The special case we wish to explicitly highlight in this regard is that of $N_{f}$ massless flavors at zero temperature. Connecting the $N_{f}=2$ theory believed to be confined, chirally broken and type (A), to a theory at sufficiently high $N_{f}>N_{f}^{c r}$ so that it is deconfined, chirally symmetric and type (C), we raise the possibility of anomalous phase [1]

$$
2<\mathrm{N}_{f}^{c}<\mathrm{N}_{f}<\mathrm{N}_{f}^{c h}=\mathrm{N}_{f}^{c r}
$$

Here $N_{f}^{c r}$ is the usual lower edge for Banks-Zaks infrared fixed point. Thus, in our scenario, this "anomalous window" precedes the conformal window. Given the results presented here, both $N_{f}=8$ and $N_{f}=12$ theories may in fact turn out to reside in the former.

Acknowledgments: We are indebted to Szabolcs Borsányi, Zoltán Fodor, Anna Hasenfratz, David Schaich and Mingyang Sun for various help. A.A. was supported by U.S. National Science Foundation under CAREER grant PHY-1151648. I.H. was supported in part by Department of Anesthesiology at the University of Kentucky.

\section{References}

[1] A. Alexandru, I. Horváth, Phys. Rev. D92 (2015) 045038, arXiv: 1502.07732.

[2] R.G. Edwards, U.M. Heller, J.E. Kiskis, R. Narayanan, Phys. Rev. D61, 074504 (2000), arXiv:hep-lat/9910041.

[3] S. Necco, Nucl. Phys. B683, 137 (2004), arXiv: hep-lat/ 0309017.

[4] A. Alexandru, I. Horváth, Phys. Lett. B722, 160 (2013), arXiv: 1210 . 7849.

[5] A. Alexandru, I. Horváth, Nucl. Phys. B891, 1 (2015), arXiv: 1405.2968.

[6] Y. Aoki et al., Nature 443, 675 (2006), hep-lat/ 0611014.

[7] S. Borsányi et al., JHEP 1009:073 (2010), arXiv: 1005. 3508.

[8] V. Dick et al., arXiv:1502.06190.

[9] A. Alexandru, I. Horváth, arXiv:1412.1777. 